\title{
Melatonin: Therapeutic Intervention in Mild Cognitive Impairment and Alzheimer Disease
}

John K. Grandy ${ }^{1,2 *}$

${ }^{1}$ Whitestone Consulting LLC- clinical contractor at Fort Drum N.Y.

${ }^{2}$ North Country Urgent Care, Watertown, N.Y.

\begin{abstract}
This article investigates the potential improvements in the neurologic patient care by utilizing melatonin as a therapeutic intervention. Melatonin is known to be involved in the circadian sleep cycle, but it has other beneficial properties e.g. antioxidant and neuroprotective. In addition, melatonin has been shown to decrease levels of soluble $\beta$-amyloid, which is known to form senile plaques that are found the brains of patients with Alzheimer disease. In this article we review melatonin's physiological activities and evidence-based therapeutic interventions in patients with mild cognitive impairment and Alzheimer disease. It was found that melatonin does not reverse the dementia symptoms in the final phases of Alzheimer disease but there was improvement in sleep-wake abnormalities, circadian rhythm disturbances, and sundown syndrome in these patients. Melatonin therapy was also seen to improve sleep quality, cognitive performance, memory, and depression in patients with mild cognitive impairment. Although melatonin therapy does not appear to be a potential cure for Alzheimer disease it does provide some important neurologic benefits in the appropriate patient population.
\end{abstract}

\section{Introduction}

Alzheimer disease (AD) is the most common form of dementia and much has been discovered recently in regards to the underlying neurophysiology. In early 2011, the diagnostic clinical criteria for AD were revised for the first time in 27 years and clinical reviews have been completed $[1,2]$. These guidelines recognize that $\mathrm{AD}$ is a disease that progresses in three phases and the pathological process begins decades before signs and symptoms manifest, which is the asymptomatic phase. Eventually mild symptoms emerge which cause some cognitive impairments but these symptoms are mild and do not cause significant problems in everyday life. However, as the disease progresses the symptoms become sever enough that dementia is evident.

$\mathrm{AD}$ is characterized by the progressive loss of cognitive functions and memory, but can also be accompanied by neurobehavioral manifestations. Mild cognitive impairment (MCI) is thought to be the intermediate stage between expected cognitive decline of normal aging and the more pronounced decline seen in dementia. According to the 2011 updates guidelines for $\mathrm{AD}$, the symptomatic predementia phase of $\mathrm{AD}$ is now referred to as $M C I$ due to $A D$. Table 1 lists the five criteria from the 2011 core criteria that must be met for the diagnosis of MCI due to $\mathrm{AD}$.

The two pathognomonic features of $\mathrm{AD}$ are the formation of senile plaques that are primarily composed of $\beta$-amyloid $(A \beta)$ and the presence of neurofibrillary tangles (NFTs) that are composed of hyperphosporlated tau proteins. Overtime there is the manifestation of gross loss of brain volume in $\mathrm{AD}$ that is associated with these two abnormal neurophysiologic features. Of course this is a simplification of the extremely complicated pathophysiological process of $\mathrm{AD}$.

Abnormal accumulations of $A \beta$ begin decades before symptoms occur and have been associated with mutations in three main genes: APP, PSEN1, and PSEN2. In earlier studies it was found that a point mutation in the APP gene on chromosome 21 and double mutations in exon 16 were pathogenic mutations associated with $\mathrm{AD}[3,4]$. Currently there are many more mutations in the APP gene that are under investigation for this gene's correlation to AD. Mutations in both PSEN1 and PSEN2 are also correlated with AD [5]. Due to these neurogenetic associations there are vehement and optimistic efforts to use the presence of these genetic mutations to diagnose $\mathrm{AD}$ during the asymptomatic preclinical phase [6].

Research suggests that the apolipoprotein E- $\varepsilon 4 / 4$ genotype (APOE- $\varepsilon 4)$ is associated with late-onset familial and sporadic AD. Carriers of the APOE- $\varepsilon 4$ allele show an increased risk for developing $\mathrm{AD}$ in a gene dose dependent manner [7]. The APOE- 44 allele is present in about $60 \%$ of $\mathrm{AD}$ patients and this allele's presence reduces the median age of onset to 68 as opposed to 84 in non-carriers [8]. A recent study demonstrated that APOE- $\varepsilon 4$ directly damages blood vessels in the blood brain barrier and that this neurovascular damage decreases $A \beta$ clearance from the brain [9].

Mental impairment and circadian disturbances e.g. sleepwake rhythm disruption are common in $\mathrm{AD}$ patients. Decreases in melatonin levels have been confirmed postmortem in patients with $\mathrm{AD}$ compared to age-matched controls [10]. The apolipoprotein E- $\varepsilon 4 / 4$ genotype is associated with considerably larger decrease in melatonin levels in this postmortem study. It has also been suggested that decreases in melatonin in $\mathrm{AD}$ is due in part to a dysfunction of noradrenergic regulation, which is accompanied by an increase in monoamine oxidase A and a resulting depletion of serotonin. This was seen in the preclinical and clinical stages of $\mathrm{AD}$ [11]. Serotonin levels are significant as it is enzymatically converted into melatonin in the pineal gland (Figure 1). Consequently, there is an association between the APOE- $\varepsilon 4$ genotype and decreases in melatonin production in some populations of patients with $\mathrm{AD}$.

*Corresponding author: John K. Grandy, Deparment of Neurophysiology, University of Buffalo, New York, USA, E-mail: khyber_john@yahoo.com

Received January 29, 2013; Accepted March 27, 2013; Published April 01, 2013

Citation: Grandy JK (2013) Melatonin: Therapeutic Intervention in Mild Cognitive Impairment and Alzheimer Disease. J Neurol Neurophysiol 4: 148 doi:10.4172/2155-9562.1000148

Copyright: @ 2013 Grandy JK. This is an open-access article distributed under the terms of the Creative Commons Attribution License, which permits unrestricted use, distribution, and reproduction in any medium, provided the original author and source are credited. 
Citation: Grandy JK (2013) Melatonin: Therapeutic Intervention in Mild Cognitive Impairment and Alzheimer Disease. J Neurol Neurophysiol 4: 148. doi:10.4172/2155-9562.1000148

1) Concern regarding change in cognition: This information should be compared with previous levels of functioning and obtained from the patient, a reliable informant, and/or clinical observation.

2) Impairment in one or more cognitive domains that is greater than expected for the patient's age and educational background: These features include memory, executive function, attention, language, and visuospatial skills. Impairment in the ability to learn and retain information (episodic memory) is more common in $\mathrm{MCl}$ patients who progress to $\mathrm{AD}$ dementia than in $\mathrm{MCl}$ patients who do not progress to $\mathrm{AD}$ dementia.

3) Preservation of independence in functional abilities: A patient with $\mathrm{MCl}$ has the functional ability to perform complex tasks, such as paying bills, preparing meals, or shopping, but demonstrates mild difficulties. For example, the person takes more time to perform these tasks, shows a decrease in efficiency, and/or has an increase in errors.

4) Failure to meet the criteria for dementia: The decline in cognitive abilities must be mild, with evidence of intraindividual change, and pose no significant social or occupational impairment.

5) Etiology of $\mathrm{MCl}$ is consistent with $\mathrm{AD}$ pathophysiologic process: For a patient to fulfill the 2011 core criteria for $\mathrm{MCl}$ other systemic causes, medical conditions, or neurologic diseases must be ruled out.

Table 1: The five criteria for $\mathrm{MCl}$ due to $\mathrm{AD}$.
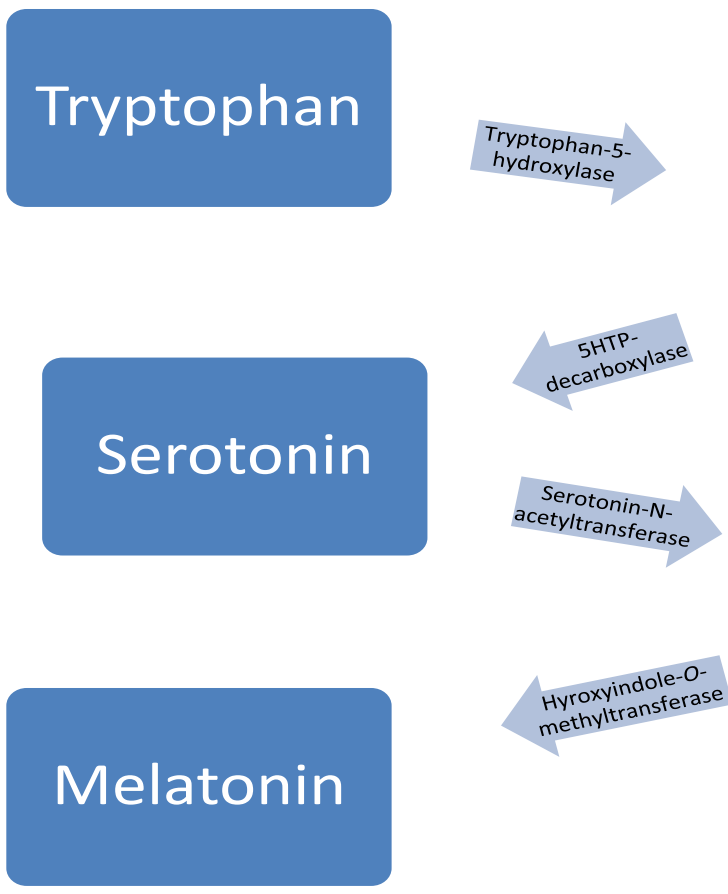
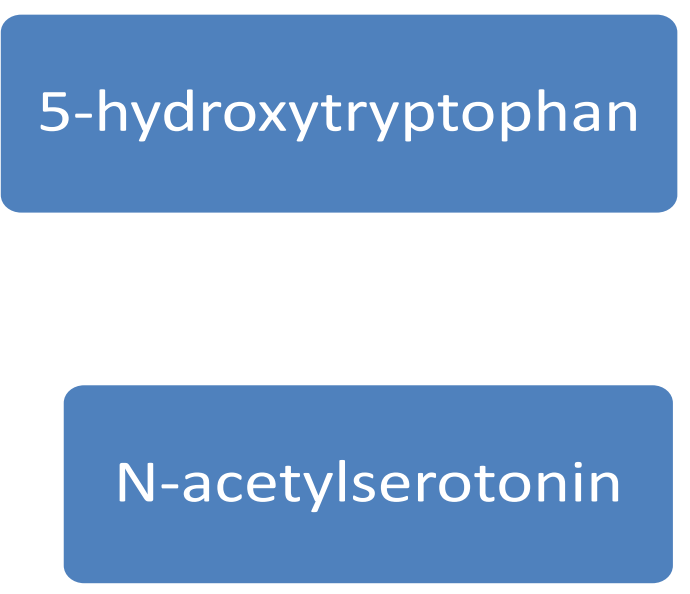

Figure 1: Melatonin synthesis from tryptophan.

\section{Methods}

All the information gathered for this article was obtained by individual searches on PubMed (http://www.ncbi.nlm.nih.gov/ pubmed). All relevant searches were completed between August 2, 2012 and August 15, 2012.

The first section focuses on the biochemical, antioxidant, and antiamyloidogenic properties of melatonin. The searches were specific to the keywords "melatonin", "function", "antioxidant", and "antiamyloidogenic". The results were limited to publications no older than January 2010. Additional references that were older than this were later added as supporting citations. Articles were selected from the search results that provided evidence of direct neurophysiological benefits of melatonin.

The second section focuses on clinical trials involving melatonin therapy in patient populations diagnosed with $\mathrm{AD}$ and MCI. The searches were specific to the keywords "melatonin", "Alzheimer disease", and "mild cognitive impairment". The results were limited to publications no older than January 2010. Additional references that were older than this were later added as supporting citations. Articles were selected from the search results that conducted studies on MCI and $\mathrm{AD}$ patient populations with sleep-wake abnormalities, circadian rhythm disturbances, and sundown syndrome.

\section{Melatonin: Structure, Function, and Synthesis}

Structurally, melatonin (N-acetyl-5-methoxytryptamine) is an indoleamine that is produced by almost every unicellular and multicellular organism, including plants and animals. Indoleamines are indole derivatives that contain a primary, secondary, or tertiary amine group.

In humans the function of melatonin is well known to be active in the synchronization of the circadian rhythm, stimulation of the immune system, seasonal reproductive regulation, and blood pressure regulation. There is a fair amount of evidence in the medical literature 
Citation: Grandy JK (2013) Melatonin: Therapeutic Intervention in Mild Cognitive Impairment and Alzheimer Disease. J Neurol Neurophysiol 4: 148. doi:10.4172/2155-9562.1000148

that demonstrates that melatonin and melatonin agonists (e.g. agomelatine) improves depression and seasonal affective disorder [12]. This would suggest that melatonin has an effect on mood.

Melatonin is synthesized from the essential amino acid tryptophan [13]. This takes place in a four step reaction (Figure 1). The first step involves the conversion of tryptophan to 5HTP (5-hydroxytryptophan) by the catalytic enzyme tryptophan-5-hydroxylase, which adds a hydroxyl group (-OH). Second, $5 \mathrm{HTP}$ is then converted into serotonin (5-hydroxytryptamine) by 5HTP-decarboxylase, which removes a carbon dioxide molecule $\left(\mathrm{CO}_{2}\right)$. In the third step serotonin enters the pineal gland where it is converted into NAS ( $\mathrm{N}$-acetylserotonin) by serotonin- $\mathrm{N}$-acetyltransferase, which adds an acetyl group $\left(-\mathrm{COCH}_{3}\right)$. NAS remains in the pineal gland and in the final phase it is converted into melatonin by hydroxyindole-O-methyltransferase, which adds a methyl group $\left(-\mathrm{CH}_{3}\right)$. Next, we will discuss melatonin's antiamyloidogenic and neuroprotective properties.

\section{Antioxidant and Neuroprotective Properties of Melatonin}

It is well established that melatonin acts as an antioxidant [14]. More importantly, it combats neurotoxins that cause molecular damage to neurons. Melatonin does this by three main mechanisms of action:

1) The inhibition of nitric oxide synthase. Nitric oxide synthase produces the free radical nitric oxide.

2) Stimulates the production of antioxidative enzymes- most important among these is glutathione.

3) Reduces free radical production at the level of the mitochondriathis process known as radical avoidance.

Prolonged exposure to free radicals and neurotoxins can cause neuropsychiatric manifestations, motor and sensory disturbances, and cognitive decline. This is primarily due to the destruction that free radicals inflict on the neurons. Recent research has demonstrated that melatonin enhances DNA repair capacity in some cells by affecting several important genes that are involved in the DNA damage responsive pathways [15]. In the R. Lui et al. study, there were $>100$ genes that were identified that are effected by melatonin after methylmethanesulfonate exposure (which causes DNA mutagen damage). This suggests possible genetic pathways in where melatonin counteracts the activities of free radicals and enhances DNA repair. Consequently, melatonin plays a pivotal role in antioxidant activity and provides neuron protection.

\section{Amyloid Accumulation and the Anti-amyloidogenic Properties of Melatonin}

Melatonin has neuroprotective and antioxidant functions in the brain. It also has demonstrated anti-amyloidogenic properties [16] These anti-amyloidogenic properties allow melatonin to decrease $A \beta$ in the brain. This is important as it was mentioned previously that $A \beta$ accumulation is one of the pathognomonic features of AD. I will now briefly discuss how $A \beta$ is formed in the brain. Keep in mind that this is a more complex neurophysiological process but a basic description will be given in order to appreciate the anti-amyloidogenic properties of melatonin.

The intregal membrane protein, amyloid precursor protein (APP), undergoes proteolysis by BACE- 1 and then by $\gamma$-secretase [17]. This is referred to as the amyloidogenic pathway (as opposed to the nonamyloidogenic pathway) as it produces $A \beta$ isoforms 39-42. $A \beta$ is continuously formed from the neuronal cell membrane when neurons conduct an impulse. Hence, $A \beta$ is a natural byproduct of neuron activity, but at high levels leads to depressed activity of the neuron [18]. This is thought to be a feedback loop that prevents neuron hyperactivity. After neuron firing $A \beta$ is broken down by neprilysin [19]. In AD pathology there is an imbalance in the production and clearance of $\mathrm{A} \beta$.

Neurotoxic forms of $A \beta$ can be produced by improper proteolytic cleavage by $\gamma$-secretase, which is a protein complex that consists of four proteins (Table 2 ). The most neurotoxic form of amyloid is $\beta$-amyloid 42 (A $\beta 42)$ [18]. Due to the hydrophobic nature of $A \beta$ (especially $\mathrm{A} \beta 42$ ), it has the propensity to form $\beta$-pleated sheets, which then forms senile plaques in the brain. These plaques can produce aggregations of peptides that form amyloid fibrils (fibrillar forms of $A \beta$ ), which are also neurotoxic [20]. High levels of fibrillary forms of $A \beta$ are also associated with loss of synapse integrity, impairment of neuron functioning, and gross loss of neurons [21]. Therefore, it can be seen that $A \beta$ properties can contribute to the abnormal neurophysiology seen in $\mathrm{AD}$.

It has long been proposed that the accumulation of $A \beta$ initiates the hyperphosphorylation of tau by interacting with metal ions. This was originally proposed in the metal hypothesis of $\mathrm{AD}$ [22] and more recently revised as the zinc dyshomeostatsis hypothesis of AD [23]. Tau and microtubule-associated proteins (MAPs) promote microtubule assembly and stability. However, hyperphosphorylated tau detaches from the neuron and MAPs, and then self-aggregates into paired helical filaments that result in NFTs [24]. The microtubule-binding domain of tau is made up of four repeating sequences (R1-R4), which tend to be flanked by the paired helical filaments of hyperphosphorylated tau. This causes microtubule destabilization and impaired axonal transport. Eventually the neuron falls apart. Therefore, in addition to being one of the pathognomonic features of $\mathrm{AD}, \mathrm{A} \beta$ may be responsible for causing the second pathognomonic feature of $\mathrm{AD}$, i.e. it initiates one of the processes that results in the presence of NFTs.

The anti-amyloidogenic properties of melatonin have been

\section{Table two: The y-Secretase Complex}

The $\mathrm{y}$-secretase is a complex of 4 proteins:

1) Presenilin-1 (PSEN1)

2) Presenilin enhancer-2 (PSEN2)

3) Nicastrin

4) Anterior pharynx-defective $1(\mathrm{APH}-1)$

The production of $A \beta$ isoforms (39-42) takes place during the amyloidogenic pathway in which the intregal membrane protein APP undergoes proteolysis in two steps: Step one: The $\beta$-site amyloid precursor protein enzyme- BACE-1 removes (proteolysis) the sAPP ${ }_{\beta}$ portion of APP while the resulting protein remains positioned in the neuron cell membrane.

Step two: Regulated intramembranous proteolysis is performed by $\gamma$-secretase. This step releases A $\beta$ isoforms (39-42) from the neuronal cell membrane.

There are two presenilin proteins in the $y$-secretase complex. This is why mutations in these two presenilin genes are strongly correlated with AD. It is proposed that the mutations can cause the production of abnormal versions of these two proteins which leads to improper proteolytic cleavage by the $\mathrm{Y}$-secretase complex. 
reviewed. The supporting evidence indicates that melatonin actively decreases levels of soluble plaque-forming $A \beta$ by interfering with the full maturation of $A \beta$. It is proposed that melatonin accomplishes this by decreasing the toxicity of $A \beta$ by making it more prone to proteolytic degradation. This would enable melatonin to inhibit $\beta$-sheet formation by preventing fibrillary amyloid from interacting with both $A \beta 40$ and $A \beta 42$. However, in one study melatonin failed to exert anti-amyloidogenic benefits after the deposition of $A \beta$ occurs [25]. This is significant pathologically as it indicates the need for earlier intervention with melatonin as abnormal accumulations of $A \beta$ appear to not only impair the normal function of neurons and to facilitate their destruction but also it has been proposed that $A \beta$ may initiate the formation of hyperphosphorylated tau. Thus initiating therapeutic intervention with melatonin before this occurs would be most prudent.

\section{Therapeutic Interventions with Melatonin}

At this juncture we have verified several neurologic benefits that are provided by melatonin: antioxidant activity, neuroprotection, and anti-amyloidogenic properties. These are direct benefits of melatonin therapy that work on a biochemical scale. However, what are some of the indirect benefits of melatonin and what patient populations have been shown to have neurologic benefits from treatment with melatonin?

There have been several studies and reviews performed on evaluating the effectiveness of melatonin in $\mathrm{AD}$ patients with sleep disorders and in patients with mild cognitive impairment. However, no general consent has been established in the medical community. A recent review has enumerated several good studies that provide sound reasoning to initiate melatonin as an add-on therapeutic tool in AD patients with sleep-wake abnormalities, circadian rhythm disturbances, sundown syndrome, and also in patients with MCI [26]. Next I will review some of the therapeutic interventions in these patient populations.

\section{Sleep-wake abnormalities, circadian rhythm disturbances, and sundown syndrome}

In $\mathrm{AD}$ patients sleep-wake abnormalities and circadian rhythm disturbances can become more pronounced as the disease progresses. Sleep disturbances are generally associated with increases in memory and cognitive impairment in patients with $\mathrm{AD}$ [27]. Treatment with melatonin has been shown to improve REM sleep and can improve some $\mathrm{AD}$ symptoms by supporting the restorative phases of the sleep cycle, which was associated with improvements in memory and cognitive impairments [26]. These clinical improvements are indirect benefits of melatonin use.

Patients with $\mathrm{AD}$ can also display symptoms e.g. confusion, anxiety, disorientation, pacing, wandering, agitation, aggression, resistance to redirection, screaming, and yelling, which are collectively referred to as sundown syndrome. AD patients can normally have these types of symptoms, but what distinguishes them from sundown syndrome is that these symptoms occur specifically in the late afternoon, evening, or at night. Keep in mind that sundown syndrome is a descriptive term used for the chronobiological phenomenon and is not an actual diagnosis. Sundown syndrome can be observed in conjunction with sleep-wake abnormalities and circadian rhythm disturbances.

Studies as early as 1997 showed improvements the in variability in sleep onset and sundown syndrome in $\mathrm{AD}$ patients with the administration of melatonin. In one study 7 out of 10 patients showed improvement after taking melatonin 3mg PO QHS for 21 days [28].
Another study using higher doses of melatonin, 6-9mg, demonstrated improvement in sundown syndrome in 12 out of $14 \mathrm{AD}$ patients with co-morbid sleep disorders [29]. Therefore, melatonin has been shown; at least in some small trials, to benefit $\mathrm{AD}$ patients with sleep disorders and sundown syndrome. However, there is conflicting data and no conclusive dose-related effectiveness established in the medical literature [30]. In addition, all the studies mentioned were done prior to the 2011 updated AD clinical diagnostic guidelines, thus raising the question as to whether or not all of the patients in the previous studies had an accurate diagnosis of AD.

\section{Mild cognitive impairment}

One of the first studies involving the use of melatonin in MCI patients was conducted in 1998 [31]. The results displayed improvements in rest-activity rhythm, sleep quality, the ability to remember previously learned items, and reductions in reported depression in these patients. A more current study, which was retrospective, demonstrated that $\mathrm{MCI}$ patients treated with melatonin 3-9mg daily for 9-18 months improved on the Mini Mental Status Exam (MMSE) and the Alzheimer's Disease Assessment Scale cognitive subscale scores [32]. In addition, Beck Depression Inventory scores showed a decrease in these same patients, indicating that melatonin may augment antidepressant therapy.

Based on these studies and the review published by Cardinali et al. there appears to be some compelling data to initiate the use of melatonin in MCI patients and even more reason to use it in $M C I d u e$ to $A D$ as there is the pathological indication that the progression of $A \beta$ plaque formation may be stymied [16]. In addition, treatment with melatonin in $\mathrm{AD}$ patients with co-morbid sleep-wake abnormalities, circadian rhythm disturbances, and sundown syndrome also seen some improvement in neurologic symptoms, but there is a lack of general consent amid the medical community.

\section{What Patients are more likely to have Neurologic Benefits from Melatonin Therapy?}

There are no current studies that demonstrate any added benefit or improvement with melatonin therapy in $\mathrm{AD}$ patients after the disease progresses to plaque formation with the manifestations of more severe dementia symptoms. Therefore melatonin is not a potential cure of $\mathrm{AD}$. However, there does appear to be some benefits in $\mathrm{AD}$ patients with sleep-wake abnormalities, circadian rhythm disturbances, and sundown syndrome, but the consensus on the therapeutic intervention and dose is not crystal clear.

The results that were discussed seem to support the addition of melatonin to patients with a recent onset of MCI as several of these studies demonstrated improvements in sleep quality, memory, and depression. However all of these studies that were reviewed where done prior to the 2011 clinical diagnostic updates for AD. Therefore there is no certainty as to if those patients were MCI due to AD or MCI due to other causes. Consequently, more retrospective studies need to be performed and refined based on the 2011 core criteria for MCI due to $\mathrm{AD}$ in an attempt to gain some consensus.

The presence of the apolipoprotein E- $4 / 4$ genotype has been correlated with decreases in melatonin and this variant allele is strongly correlated with the development of AD. Therefore studies using melatonin therapy involving $\mathrm{MCI}$ and $\mathrm{AD}$ patients with the APOE- $\varepsilon 4$ genotype are warranted. However, there are no current inexpensive routine clinical tests available to screen for this genotype. This highlights the potential importance of affordable neurogenetic testing in the future as patients identified with the APOE- $\varepsilon 4$ genotype 
Citation: Grandy JK (2013) Melatonin: Therapeutic Intervention in Mild Cognitive Impairment and Alzheimer Disease. J Neurol Neurophysiol 4: 148. doi:10.4172/2155-9562.1000148

would appear to be more likely to benefit from the earlier initiation of melatonin therapy. This could be an alternative as opposed to waiting for signs and symptoms of MCI and AD dementia to manifest and then initiate treatment, which is unfortunately the current paradigm.

As previously mentioned, melatonin has not been shown to reverse severe symptoms of dementia nor is it proposed here to be a cure for $\mathrm{AD}$. The results demonstrate that there are improvements seen in MCI patients with melatonin therapy. These two findings enforce the proposal that decreases in melatonin are more likely to be a result of $\mathrm{AD}$ pathology as melatonin production is decreased after senile plaques have formed. The dementia symptoms may become more profound after the subsequent decrease in melatonin. This would support the new model of the pathophysiological process of $\mathrm{AD}$ in which the disease progresses in three different phases based on the 2011 updates.

1) A preclinical asymptomatic phase- it is proposed that accumulations of $A \beta$ plaques begin to form (and NFTs) in this phase years to decades before any obvious symptoms are observed clinically.

2) A symptomatic predementia phase (MCI due to $\mathrm{AD}$ ) - it is thought that during this phase the $A \beta$ plaques and NFTs begin to accumulate to a point where mild but not severe symptoms emerge. The patient can live a normal life without assistance but may struggle somewhat with certain tasks.

3) A dementia phase- during this phase there has been gross loss in brain volume and enough destruction to the neurons due to the underlying $\mathrm{AD}$ pathological processes that severe cognitive and behavioral symptoms are present. At this point the patient usually requires some type of assistance for daily living.

In the first and second phase of $\mathrm{AD}$ there appears to be a clinical benefit to using melatonin as a therapeutic intervention or as an addon medication. So there is an opportunity to improve the quality of patient care and to prevent the deterioration of cognitive abilities and executive skills. After the final phase of $\mathrm{AD}$ commences there appears to be no therapeutic interventions that can reverse the accumulation of $A \beta$ plaques and NFTs in the brain. However, in the dementia phase of $\mathrm{AD}$ melatonin treatment may improve sleep quality and sundown syndrome.

There may be other potential therapeutic options to co-administer along with melatonin therapy. A recent study demonstrated that nutritional intervention with an oily emulsion of docosachexaenoic acid (DHA) phospholipids containing melatonin and tryptophan resulted in an improvement in MMSE and semantic verbal fluency after 12 weeks [33]. In this study the older adults with MCI displayed a significant improvement in cognitive function with this nutritional intervention. This highlights the importance of not only melatonin supplementation, but the potential benefit of DHA and tryptophan in this patient population. The potential importance of tryptophan most likely lies in the fact that it is intrinsically involved in melatonin synthesis (Figure 1).

\section{Cautions when Administering Melatonin Therapy}

Melatonin is a product normally produced in the brain and body so there are no severe adverse drug reactions or toxicity reported at the research doses, which typically ranged from 1 to $9 \mathrm{mg}$; depending on the study. The recommended over the counter dose is usually between 3 and $5 \mathrm{mg}$ PO. Common side effects of orally ingested melatonin are daytime sleepiness, dizziness, and headaches. Additional side effects can include abdominal discomfort, confusion, mild anxiety, irritability, or depressed feelings that are short-lasting. Over the counter melatonin can interact with anticoagulants, immunosuppressants, diabetic medications, and birth control medications. Therefore it is important to review the patient's current list of medications prior to administering melatonin.

Therapeutic intervention with melatonin has demonstrated improvements in neurologic symptoms and patient care in certain patient populations. If the clinician decides to administer melatonin for MCI or co-morbid sleep disorders in the $\mathrm{AD}$ patient a few things should be considered. First, whether or not the patient stands to benefit. This requires a thorough history with a second person report and accurately determining what phase of the disease that the patient is likely to be in. This requires an understanding of the updated 2011 diagnostic clinical guidelines for AD. Secondly, there is no established dose only a recommended dose range. This can be reasonably initiated low and gradually increased. Therefore, tolerability must be considered as well as liver status as melatonin is hepaticaly metabolized. Other medications that melatonin can potentially interact with must be considered as well.

\section{References}

1. Grandy JK (2012) Updated guidelines for the diagnosis of Alzheimer disease: a clinical review. JAAPA 25: 50-55.

2. Budson AE, Solomon PR (2012) New diagnostic criteria for Alzheimer's disease and mild cognitive impairment for the practical neurologist. Pract Neurol 12: 8896.

3. Goate A, Chartier-Harlin MC, Mullan M, Brown J, Crawford F, et al. (1991) Segregation of a missense mutation in the amyloid precursor protein gene with familial Alzheimer's disease. Nature 349: 704-706.

4. Mullan M, Crawford F, Axelman K, Houlden H, Lilius L, et al. (1992) A pathogenic mutation for probable Alzheimer's disease in the APP gene at the N-terminus of beta-amyloid. Nat Genet 1: 345-347.

5. Cruts M, Hendriks L, Van Broeckhoven C (1996) The presenilin genes: a new gene family involved in Alzheimer disease pathology. Hum Mol Genet 5 Spec No: 1449-1455.

6. Grandy JK (2011) Biomarkers and gene mutations as aids for detecting AD early. JAAPA 24: 56-57.

7. Strittmatter WJ, Roses AD (1995) Apolipoprotein E and Alzheimer disease Proc Natl Acad Sci U S A 92: 4725-4727.

8. Leoni $V(2011)$ The effect of apolipoprotein $E$ (ApoE) genotype on biomarkers of amyloidogenesis, tau pathology and neurodegeneration in Alzheimer's disease. Clin Chem Lab Med 49: 375-383.

9. Bell RD, Winkler EA, Singh I, Sagare AP, Deane R, et al. (2012) Apolipoprotein E controls cerebrovascular integrity via cyclophilin A. Nature 485: 512-516.

10. Liu RY, Zhou JN, van Heerikhuize J, Hofman MA, Swaab DF (1999) Decreased Melatonin Levels in Postmortem Cerebrospinal Fluid in Relation to Aging Alzheimer's Disease, and Apolipoprotein E-Epsilon4/4 Genotype. J. Clin. Endocrinol. Metab. 84: 323-327.

11. Wu YH, Feenstra MG, Zhou JN, Liu RY, Toranõ JS, et al. (2003) Molecular changes underlying reduced pineal melatonin levels in Alzheimer disease: alterations in preclinical and clinical stages. J Clin Endocrinol Metab 88: 58985906.

12. Quera Salva MA, Hartley S, Barbot F, Alvarez JC, Lofaso F, et al. (2011) Circadian rhythms, melatonin and depression. Curr Pharm Des 17: 1459-1470.

13. Neuroendocrinology in Physiology and Medicine (2010) Edited by P. Michael Conn and Marc E. Freeman. Humana Press Inc. Totowa, New Jersey.

14. Reiter RJ, Manchester LC, Tan DX (2010) Neurotoxins: free radical mechanisms and melatonin protection. Curr Neuropharmacol 8: 194-210.

15. Liu R, Fu A, Hoffman AE, Zheng T, Zhu Y (2013) Melatonin enhances DNA repair capacity possibly by affecting genes involved in DNA damage responsive pathways. BMC Cell Biol 14: 1. 
Citation: Grandy JK (2013) Melatonin: Therapeutic Intervention in Mild Cognitive Impairment and Alzheimer Disease. J Neurol Neurophysiol 4: 148. doi:10.4172/2155-9562.1000148

16. He H, Dong W, Huang F (2010) Anti-amyloidogenic and anti-apoptotic role of melatonin in Alzheimer disease. Curr Neuropharmacol 8: 211-217.

17. Querfurth HW, LaFerla FM (2010) Alzheimer's disease. N Engl J Med 362: 329-344.

18. Haass C, Selkoe DJ (2007) Soluble protein oligomers in neurodegeneration: lessons from the Alzheimer's amyloid beta-peptide. Nat Rev Mol Cell Biol 8 : $101-112$.

19. Yasojima K, Mc Geer EG, Mc Geer PL (2001) Relationship between beta amyloid peptide generating molecules and neprilysin in Alzheimer disease and normal brain. Brain Res. 919: 115-121.

20. Alvarez A, Alarcón R, Opazo C, Campos EO, Muñoz FJ, et al. (1998) Stable complexes involving acetylcholinesterase and amyloid-beta peptide change the biochemical properties of the enzyme and increase the neurotoxicity of Alzheimer's fibrils. J Neurosci 18: 3213-3223.

21. Iwata N, Tsubuki S, Takaki Y, Watanabe K, Sekiguchi M, et al. (2000) Identification of the major Abeta1-42-degrading catabolic pathway in brain parenchyma: suppression leads to biochemical and pathological deposition. Nat Med 6: 143-150.

22. Bush Al, Tanzi RE (2008) Therapeutics for Alzheimer's disease based on the metal hypothesis. Neurotherapeutics 5: 421-432.

23. Craddock TJ, Tuszynski JA, Chopra D, Casey N, Goldstein LE, et al. (2012) The zinc dyshomeostasis hypothesis of Alzheimer's disease. PLoS One 7 : e33552.

24. Alonso A, Zaidi T, NovakM, Grundke-lqbal I, Iqbal K(2001)Hyperphosphorylation induces self-assembly of tau into tangles of paired helical filaments/straight filaments. Proc Natl Acad Sci U S A 98: 6923-6928.
25. Quinn J, Kulhanek D, Nowlin J, Jones R, Praticò D, et al. (2005) Chronic melatonin therapy fails to alter amyloid burden or oxidative damage in old Tg2576 mice: implications for clinical trials. Brain Res 1037: 209-213.

26. Cardinali DP, Furio AM, Brusco LI (2010) Clinical aspects of melatonin intervention in Alzheimer's disease progression. Curr Neuropharmacol 8: 218 227.

27. McCurry SM, Reynolds CF, Ancoli-Israel S, Teri L, Vitiello MV (2000) Treatment of sleep disturbance in Alzheimer's disease. Sleep Med Rev 4: 603-628.

28. Fainstein I (1997) Effects of Melatonin in Elderly patients With Sleep Disturbance. A Pilot Study. Curr. Ther. Res. 58: 990-1000.

29. Brusco LI, Márquez M, Cardinali DP (2000) Melatonin treatment stabilizes chronobiologic and cognitive symptoms in Alzheimer's disease. Neuro Endocrinol Lett 21: 39-42.

30. Khachiyants N, Trinkle D, Son SJ, Kim KY (2011) Sundown syndrome in persons with dementia: an update. Psychiatry Investig 8: 275-287.

31. Jean-Louis G, von Gizycki H, Zizi F (1998) Melatonin effects on sleep, mood, and cognition in elderly with mild cognitive impairment. J Pineal Res 25: 177 183

32. Furio AM, Brusco LI, Cardinali DP (2007) Possible therapeutic value of melatonin in mild cognitive impairment: a retrospective study. J Pineal Res 43 404-409.

33. Rondanelli M, Opizzi A, Faliva M, Mozzoni M, Antoniello N, et al. (2012) Effects of a diet integration with an oily emulsion of DHA-phospholipids containing melatonin and tryptophan in elderly patients suffering from mild cognitive impairment. Nutr Neurosci 15: 46-54. 